\title{
The effect of magnetic treatment on the effectiveness of inhibition in oilfields
}

\author{
I.A.Golubev, ${ }^{l, *}$ A.B.Laptev, ${ }^{2}$ E.LAlekseeva., ${ }^{I}$ N.O.Shaposhnikov, ${ }^{I}$ A.M. Povyshev $^{l}$ and M.K.Kurakin. ${ }^{I}$ \\ 1 - Peter the Great St. Petersburg Polytechnic University, 195251 St. Petersburg, Russia \\ 2 -Federal State Unitary Enterprise All-Russian scientific research Institute of aviation materials, 105005 Moscow
}

\begin{abstract}
The article considers the possibility of increasing the efficiency of chemical reagents by treating inhibited solutions with a magnetic field. It is shown that a various method for generating the magnetic field has a different effect (Some positive some negative). The best results can be achieved with anti-scale magnetic treatment using permanent magnets. Modified inhibitors (after magnetic treatment) have enhanced protective effectby their adsorption capacity with respect to metal increases.
\end{abstract}

\section{Introduction}

Most of the oil fields being developed using oilfield water flooding technology are characterized by high water cut of the produced wells, leads to a significant increase in corrosion of technological equipment and pipelines. The main method of corrosion protection in oil fields is the treatment of oil-water environments with corrosion inhibitors. This makes it possible to improve the reliability and durability of the equipment without any significant interference in the process of collection and preparation.

The use of inhibitors reduces the aggressiveness of electrolytic media as a result of a decrease in the corrosion activity of the surface of metals. Cathode type inhibitors lead to a decrease in the ability of electron assimilation. Anode type inhibitors lead to inhibition of metal ions leaving the metal surface. The combination of the processes using the mixed type inhibitor is also possible.

After the adsorption of the inhibitor on the metal, a mono- or multimolecular adsorption film is formed, limiting the surface contact area with the corrosive media and serving as a barrier to self-dissolution processes [1].

There are two ways to improve the processing of oil-fields: creating new effective chemical reagents and improved characteristics (or parameters) of known inhibitors. The first solution is costly because it requires the completion of a whole range of work to obtain new reagents, often based on expensive raw materials. The second - makes it possible, acting on the mentioned factors, to increase the efficiency of inhibition processes.

One of the ways to influence the inhibition process is pre-treatment of oil-fields at the stage of reagent introduction by a magnetic field. As shown by numerous independent studies [2 - 5], performed in laboratory and field conditions, the magnetic field can reduce the consumption of corrosion inhibitors, while increasing the processing efficiency. However, previous work has not beenaddressed range of topics associated with the explanation of the mechanism consisting of the magnetic field on the corrosion inhibitors and development environment required for this technology.

The aim of this work is the practical testing of the method of magnetic intensification of inhibition processes, including the study of the main magnetic devices in synergy with the corrosion inhibitors used, as well as an explanation of the mechanism of this effect.

\section{Research problem statement}

Based on an analysis of the experience of using magnetic processing apparatuses in the oil industry [6], two types of installations were chosen for further research: with magnetic field sources in the form of permanent magnets and electromagnets.

a permanent magnet installation (Figure 1) consists of: a container for the treated emulsion (oil in water Designed for laboratory testing,); chambers for treating liquids with a magnetic field with permanent magnets mounted in the housing; reagent inlet and receptacle for studying samples. Reagent input is carried out by means of a peristaltic type metering pump. 


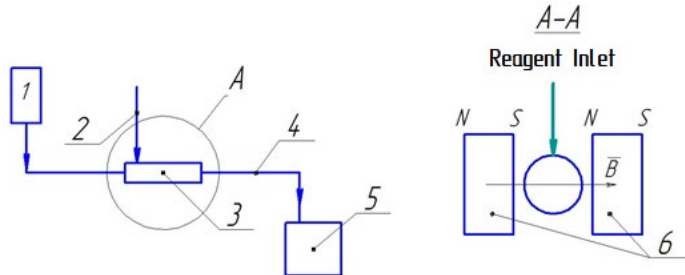
field

Fig. 1. Installation diagram with a constant magnetic

1 - emulsion tank, 2 - reagent inlet pipe, 3 - magnetic processing chamber, 4 - emulsion reagent outlet pipe, 5 electrochemical cell, 6 - permanent magnets

Permanent magnets are located at different poles opposite each other, perpendicular to the flow being processed so that the Lorentz force is directed along the reagent inlet tube [6]. By changing the polarity of the magnets, it is possible to create a zone of increased concentration of hydroxonium ions (Lorentz force up) or hydroxyl ions (Lorentz force down), as shown in Figure 1.

The designed installation with an electromagnet is a control unit (pulse generator) and a solenoid with nozzles for input and output of the processed fluid (Figure 2). The fluid flow is subjected to a treatment with a low-frequency magnetic field of a given frequency when flowing through a solenoid.

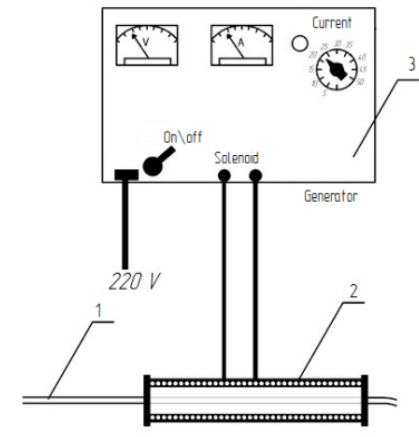

Fig. 2.Scheme of the electromagnetic installation

1 connecting tubes; 2 - solenoid; 3 - low frequency current generator with a control unit.

The results of the chemical composition analysis of the reagents actively used in the oil fields showed that amines, quaternary salts of amines, amides and imidazolines are active bases for inhibitors, and demoxifiers - ethoxylated compounds of fatty acids, esters and alcohols. Therefore, reagents on these bases have been taken for investigations.

\section{Methods of analysis}

In order to simulate inhibition processes, the emulsion treated with the reagent and magnetic field was merged into electrochemical cells. The working electrode of the cell was made of steel 20. Areference platinum electrode and a silver chloride reference electrode were used. The solution was mixed in a cell to simulate the laminar flow regime in the pipeline.

Processing was carried out by the same method for two magnetic devices in series. The effect of magnetic treatment on the effectiveness of inhibition was determined by assessing the change in the corrosion rate in untreated and treated environments.

\section{The results of processing a constant magnetic field}

In order to study the effect of a constant magnetic field, as well as a change in the direction of the magnetic induction vector, two experiments were performed on the inhibition efficiency (Figure 3).

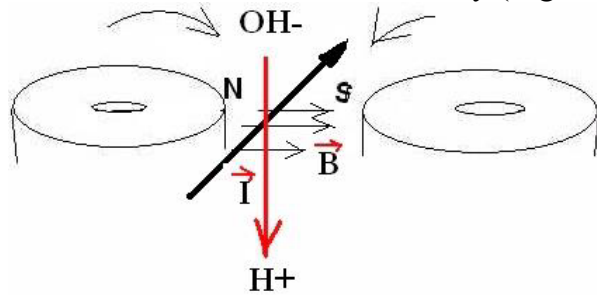

Induction current in the direction of the water layer

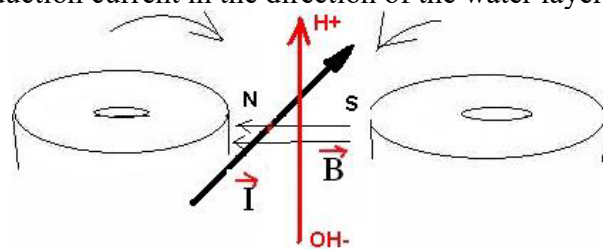

Inducing a current in the direction of the inhibitor layer

Fig. 3. Flow direction diagram

This method was developed on the basis of the principles of magnetic hydrodynamics - the theory of motion of charged particles in intersecting magnetic and electric fields [7, 8]. The method is called magnetohydrodynamic processing (MHDP). In order to obtain representative MHDP results, the tests were carried out in accordance with the methodology of the full factorial experiment. Variables were: time of magnetic action; inhibitor concentration; acidity of the medium. The measured parameter is the corrosion rate. The test results are presented in graphs (Figure $4,5)$

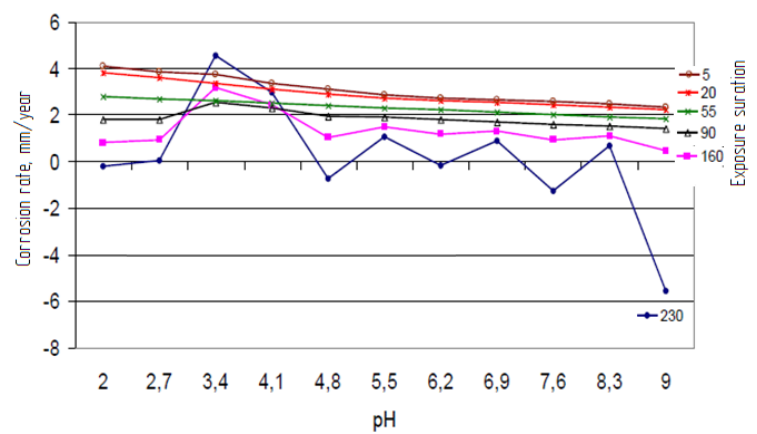

Fig.4. Change in the corrosion rate of steel with the direction of the induction vector towards the inhibitor layer. 


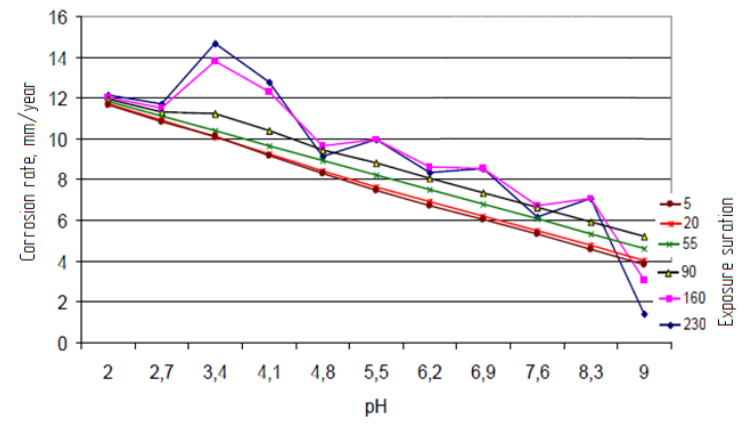

Fig. 5. Change in the corrosion rate of steel with the direction of the induction vector towards the water layer.

An analysis of the experimental results showed that when the magnetic induction vector is directed towards the input of the inhibitor, the efficiency of anticorrosion treatment increases, due to a change in the charges of ions, which increases the protective properties of the inhibitor. The Lorentz force, in this case, moves the hydroxonium ions to the input region of the reagent.

\section{The results of processing with a low-frequency electro-magnetic field during inhibition}

In order to study the effect of an electro-magnetic field on the efficiency of inhibition, tests were carried out during which the direction of the magnetic induction vector was changed as the main parameter involved in the molecular interaction of reagent particles and water at the ionic level. In a magnetic installation of this type, the magnetic induction vector is directed along the axis of the solenoid. Its direction depends on the polarity of the connection of the solenoid to the current generator.

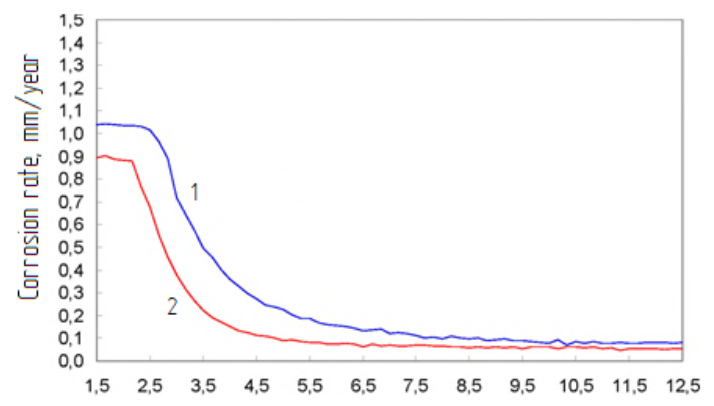

Fig. 6. Change in the corrosion rate of steel in the direction of the magnetic induction vector along the flow

1 - Inhibitor; 2 - Inhibitor + magnetic field

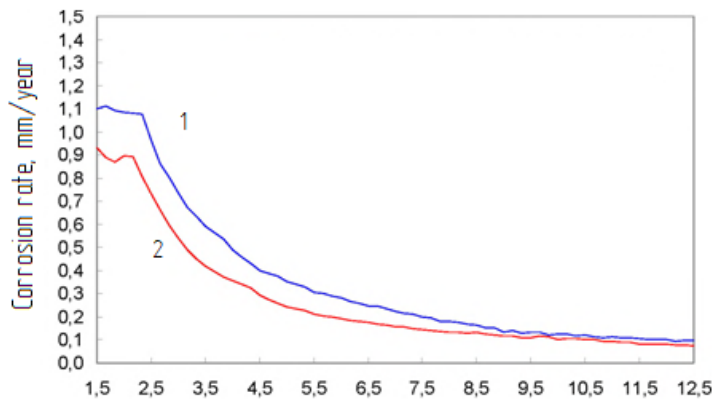

Fig. 7. Change in the corrosion rate of steel when the direction of the magnetic induction vector against the movement of the flow

1 - Inhibitor; 2 - Inhibitor + magnetic field

As can be seen from the graphs (Fig. 6, 7), as a result of treating the liquid with an alternating magnetic field together with a corrosion inhibitor, the efficiency of the latter does not significantly increase. The degree of influence of an alternating magnetic field on the corrosiveness of the medium is practically absent. Changing the direction of the induced current also did not significantly affect the processing efficiency.

\section{Analysis of the results. Identification of the mechanism of physico-chemical processing}

During the tests, two types of magnetic action were considered: a constant and an alternating magnetic field on a corrosion inhibitor. The first type of apparatus showed better results in reducing the corrosion rate than the second one. This is due, as expected, to a different mechanism of the effect of a magnetic field on reagents.

In a solenoid is creating an alternating magnetic field, the magnetic induction vector is directed along the axis along the flow of a moving fluid. The forces arising from the processing are more magnetic, electrostatic in nature, for an hour, therefore, the effect on ions does not occur and a positive effect on the inhibitors is not observed.

Inhibitors interact with the aqueous phase and the metal surface at the ionic level. The arrangement of permanent magnets with different poles opposite each other creates a magnetic field perpendicular to the flow of the medium being processed and leads to the movement of ions with different charges in opposite directions induced in a magnetic electric field. An increased concentration of hydroxonium ions forming complexes with the inhibitor is created in the reagent input area.

These complexes have an increased value of the electrostatic potential, in comparison with the standard inhibitor molecule. Such a modified inhibitor has an increased protective effect caused by increased adsorption capacity with respect to the metal.

In MHDP, a change in the composition of the corrosive medium occurs in the local volume of the 
flow; in this connection, studies are interesting [911], where local volumes with a changed composition of ions are separated. Table 1 shows the results.

Table 1. Characteristics of the flow of a corrosive medium $(0.1 \%$ sodium chloride solution) after its MHDP at various fluid speeds

\begin{tabular}{|l|l|l|l|l|l|l|}
\hline № & $\mathbf{p H}$ & $\begin{array}{l}\mathbf{E} \\
\mathbf{m},\end{array}$ & $\begin{array}{l}\text { Ecor, } \\
\mathbf{V}\end{array}$ & $\begin{array}{l}\mathbf{I c o r}, \\
\mathbf{A} / \mathbf{M}^{2}\end{array}$ & $\begin{array}{l}\mathbf{P}, \\
\mathbf{m m} \\
\mathbf{y e a r}\end{array}$ & $\begin{array}{l}\mathbf{K}, \\
\mathbf{g} / \mathbf{m}^{2} \\
\mathbf{h}\end{array}$ \\
\hline $\mathbf{1}$ & 3,6 & 210 & $-0,75$ & 2,3 & 2,7 & 2,43 \\
\hline $\mathbf{2}$ & $\begin{array}{l}3,67 \\
8\end{array}$ & $\begin{array}{l}207, \\
4\end{array}$ & $-0,809$ & 1,9 & 2,2 & 1,7 \\
\hline $\mathbf{3}$ & $\begin{array}{l}4,49 \\
6\end{array}$ & $\begin{array}{l}158, \\
1\end{array}$ & $-0,891$ & 0,8 & 1,7 & 0,92 \\
\hline $\mathbf{4}$ & $\begin{array}{l}5,38 \\
0\end{array}$ & $\begin{array}{l}108, \\
7\end{array}$ & $-0,92$ & 0,3 & 0,4 & 0,31 \\
\hline $\mathbf{5}$ & $\begin{array}{l}6,34 \\
3\end{array}$ & 52,0 & $-0,998$ & 0,19 & 0,13 & $\begin{array}{l}0,18 \\
5\end{array}$ \\
\hline $\mathbf{6}$ & $\begin{array}{l}7,01 \\
3\end{array}$ & 14,4 & $-1,002$ & 0,15 & 0,09 & 0,13 \\
& & & & & 7 \\
\hline
\end{tabular}

From table 1 it is seen that the use of a magnetic field significantly changes the electrochemical characteristics of the medium. Given that, in accordance with the Nernst equation for a particular case of the system, the metal is an $\mathrm{M}$-ion $\mathrm{Mz}+(\mathrm{z}-$ charge number of metal ions in a solution) in the form:

\section{$\mathrm{E}=\mathrm{E}_{0}+(\mathrm{RT} / \mathrm{zF}) \operatorname{lnaM}$}

Where R - gas constant; T - temperature, $\mathrm{K}$; aM activity of metal ions; $\mathrm{Z}$ - number of electrons involved in the reaction; F - Faraday number.

Redox electrodes consist of an inert metal (for example, $\mathrm{Pt}$ ), immersed in a solution containing both oxidized (Ox) and reduced (Red) forms of the substance: Ox, Red $\mid \mathrm{Pt}$; Ox $+\mathrm{z}$ e $-\leftrightarrow$ Red;

$$
\mathrm{E}_{\mathrm{h}}=\mathrm{E}_{\mathrm{h} 0}+(\mathrm{RT} / \mathrm{zF}) \ln (\mathrm{a}(\mathrm{Ox}) / \mathrm{a}(\mathrm{Red})
$$

Here a $(\mathrm{Ox})$ is the activity of oxidizing agents

a (Red) - activity of reducing agents

Thus, the change in the redox potential in the MHDP $\Lambda$ Eh process is a change in the potential in the medium and for the metal potential:

$$
\Lambda \mathrm{E}=\mathrm{E}_{0}+\Lambda \mathrm{E}_{\mathrm{h}}
$$

At very small values of the anodic current density (approximately for $\mathrm{ia}<10^{-2} \mathrm{~A} / \mathrm{m}^{2}$, the dependence of the metal ionization overvoltage on the anodic current density can be expressed by the linear equation:

$$
\Delta \mathrm{E}=\mathrm{kia},
$$

( $\mathrm{k}$ - coefficient depending on the metal, the composition of the corrosion temperature, etc.).

Or the change in current of the anode component of the corrosion process can be expressed:

$$
\Delta \mathrm{ia}=\left(\mathrm{E}_{0}+\Lambda \mathrm{E}_{\mathrm{h}}\right) / \mathrm{k}
$$

Thus, a change in the redox potential directly affects the change in the corrosion rate in the medium of a specific composition. In turn, the redox potential of a corrosive medium can be influenced by using a magnetic field. With a change in the electrochemical characteristics of a corrosive medium in a magnetic field, the aggressiveness of the medium changes, the effectiveness of corrosion inhibitors, and many other effects are observed.

In conclusion, it should be noted that using MHDP of formation water of oil and gas fields is a promising way to reduce the corrosion activity of formation water and increase the effectiveness of corrosion inhibitors.

\section{References}

1. Rakhmankulov D.L. Corrosion inhibitors. Vol. 1. Fundamentals of the theory and practice of application / Rakhmankulov D.L., Bugai D.E., Gabitov A.I., Golubev M.V., Laptev A.B., Kalimullin A.A. - Ufa: State publishing house of scientific and technical literature "Reaktiv", 1997. - 296 p.

2. Lesin V.I. The influence of the magnetic field on the properties of substances [Electronic resource]: What happens to liquid solutions after they are processed by a magnetic field // URL: http://magneticliquid.narod.ru/autority/092.htm

3. Inyushin N.V., Ishemguzhin E.I. and other devices for magnetic processing. Ufa: State Publishing House of Scientific and Technical Literature Reaktiv, 2000.

4. Khasanov M.M., Ragulin V.V., Mikhailov A.G., Shaydakov V.V., Nikitin R.V., Laptev A.B., Knyazev V.N. The effect of a magnetic field on deposits of carbonate sediments in wells [Electronic resource]: "Oil and Gas Business", 2002. No. 2 // URL: http://ogbus.ru/authors/ Khasanov / Khasanov_1.pdf

5. Golubev Ivan Quality improvement of oilcontaminated wastewater, meant for injection into formation, using two-stage treatment technology / Golubev Ivan, Karpova Yana / J. Ecol. Eng. 2017; 18(3):48-52

6. Laptev A.B. Methods and aggregates for magnetohydrodynamic processing of oil-water media. dissertation for the degree of Doctor of Technical Sciences / GOUVPO "Ufa State Petroleum Technical University". Ufa, 2008

7. Gogolev D.A. Improving the efficiency of the processes of inhibition and demulsification of oilwater environments through their magnetohydrodynamic treatment: dis. ... cand. tech. sciences. - Ufa, 2006.

8. Belsky A. A., Kupavykh K. S., Sandyga M. S., Morenov V. A., Wind Turbine Electrical Energy Supply System for Oil Well Heating/ Energetika. Proceedings of CIS higher education institutions and power engineering associations" ("Izvestiya Vysshikh Uchebnykh Zavedenii, Energetika"), № 2, T 62, 2019. C 146 - 154.

9. Tusenkov A.S., Kononov D.V., Bugai D.E., Laptev A.B. Changes in the corrosiveness of water during the transport of oil-water mixture through a 
lined pipeline. Electronic scientific journal Oil and Gas Business. 2011. No 5. S. 89-95.

10. Tusenkov A.C., Kononov D.V., Bugai D.E., Laptev A.B. Assessment of the possibility of using a surfactant to reduce oil acidification when pumping oil-water emulsion through pipelines. Oil and gas business. 2011. T. 9. No. 2. P. 38-40.

11. Kononov D.V., Laptev A.B., AkhiyarovR.Zh., Bugai D.E. Improving the safety of operation of water circulating systems of industrial enterprises by regulating the ionic composition of the aquatic environment. Problems of collection, preparation and transportation of oil and oil products. 2013. No. 2 (92). P. 100-106. 